\title{
Overview of Optimal Breast-feeding Practices and a Study of Breast-feeding Practices among Suburban Mothers in South India
}

\author{
Edwin Dias and Kumar B \\ Department of Paediatrics, Srinivas Institute of Medical Sciences and Research Centre, \\ Mangalore. India \\ E-mail:dredwindias@gmail.com
}

Type of the Paper: Research Article.

Type of Review: Peer Reviewed.

Indexed In: OpenAIRE.

DOI: http://dx.doi.org/10.5281/zenodo.572987.

Google Scholar Citation: $\underline{\text { IJHSP}}$

\section{How to Cite this Paper:}

Dias, Edwin., \& B., Kumar. (2017). Overview of Optimal Breast-feeding Practices and a Study of Breast-feeding Practices among Suburban Mothers in South India. International Journal of Health Sciences and Pharmacy (IJHSP), 1(1), 5-11.

DOI: http://dx.doi.org/10.5281/zenodo.572987.

International Journal of Health Sciences and Pharmacy (IJHSP)

A Refereed International Journal of Srinivas University, India.

(C) With Authors.

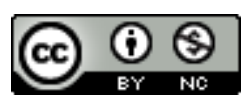

This work is licensed under a Creative Commons Attribution-Non Commercial 4.0 International License subject to proper citation to the publication source of the work.

Disclaimer: The scholarly papers as reviewed and published by the Srinivas Publications (S.P.), India are the views and opinions of their respective authors and are not the views or opinions of the SP. The SP disclaims of any harm or loss caused due to the published content to any party. 


\title{
Overview of Optimal Breast-feeding Practices and a Study of Breast-feeding Practices among Suburban Mothers in South India
}

\author{
Edwin Dias and Kumar B \\ Department of Paediatrics, Srinivas Institute of Medical Sciences and Research Centre, \\ Mangalore. India \\ E-mail:dredwindias@gmail.com
}

\begin{abstract}
Background: The status of breast milk practices is dismal in India in spite of specialized nutrient to newborns by way of breast milk. Culture influences breastfeeding decisions of mothers which have an influence on overall growth, development, health and nourishment of infants and children. Objectives: Assessing the maternal information, approach, and practices towards breastfeeding among mothers. Breastfeeding correlation with a number of antenatal visits, hospital education, maternal education, parity, mode of delivery, holding the baby after delivery, socioeconomic status, employment, religion and sex of the child, and prelacteal feeds. Methods: In a study, 400 mothers with children up to 2 years attending hospitals over a one year period. Data was collected using a self-administered standardized questionnaire. Statistical Analysis: The data was analysed regarding mean, frequencies, percentages, Chi-Square test and Spearman's correlation test. Results: Breast feeding was deferred in mothers who delivered by Caesarean section due to mothers being kept for observation. Forty-three point eight \% of mothers initiated first breast feeds within an hour. Eighty-eight point six \% had initiated breastfeeds within one to four hours, however, it was. Prelacteal feeds were given by twenty-seven \% mothers. Most mothers fed colostrum to the infants. Mean duration of exclusive breastfeeding was four and a half months. There was no association between duration of exclusive breastfeeding with parity, hospital education, holding of baby socioeconomic status and sex of the child. 34\% of mothers administered in addition to breast feeding bottle-feeding. The mean age of starting complementary foods was 6 and half months. Conclusion: Due to cultural factors and other social promotional activities, the majority of the mothers had adequate knowledge and a positive attitude towards infant breastfeeding practice. Delay in the initiation of breastfeeding will impact education programs and neonatal feeding practices.
\end{abstract}

Keywords: Breastfeeding, Knowledge Practices, Prelacteal feeds, Colostrum.

\section{INTRODUCTION :}

Breastfeeding is provided young infants with the nutrients they need for vigorous growth and development. Breast feeding is the natural way of feeding for healthy infants easy to digest, always available, comes at the right feeding temperature, and carries little infectious diseases, particularly with respect to diarrhoea encourages close body contact between mother and infant offers adequate nutrition during first 6 months of life. Complementary breastfeeding should not discourage continuation of breast feeding. Ideally, breast feeding practice includes that the baby should be attached to the breast within the first hour of birth, whenever possible. Particularly during the first days of birth the mother should ask for help, support, and practical advice how to position the baby. The child should be turned towards the mother with its whole body and take not only the nipple but a larger portion of the breast into its mouth. Breastfeeding can also be performed after a caesarean section. To promote the formation of breast milk the baby should 
suckle on both breasts during the first days. A larger amount of milk will be produced after a few days of birth. Temporary supplementary feeding with water or other liquids should be offered if it is deemed necessary by the pediatrician. Breastfeeding promotes the gradual loss of the mother's body weight and the extra body fat stores that were deposited during pregnancy. The amount of fat loss increases with the duration of full breastfeeding. Additional active weight reduction during breastfeeding with the use of restrictive diets is not recommended because it may have undesirable effects on milk composition. As breast milk composition is being researched, more and more factors are discovered in it with beneficial attributes. Most of our knowledge on breast milk cells comes from studies of leucocytic populations, which often are the dominant cells in colostrum and early lactation milk. However, in mature human milk, leucocytes are usually found in low numbers and the dominant cells are of epithelial origin. Appropriate breastfeeding is crucial for the optimal development of the infant. Breast milk encourages sensory and cognitive development and safeguards the infant against infectious and chronic diseases. Exclusive breastfeeding diminishes infant mortality due to common childhood diseases such as diarrhea, pneumonia, and assists infant for earlier recovery during illness [1].

In 2016 IMR is 48 per 1000 live births in India each year and two-thirds of these are related to unsuitable infant feeding practices. In India, one survey reveals that only 23.4 percent children under three years are breastfed within an hour of birth and only 46.3 percent children in the age group 0-5 months are exclusively breastfed [2]. In India, breastfeeding in suburban areas are molded by the beliefs of a community, and by social, cultural and educational factors. Effective breastfeeding hinges on, not only on the mother's education but also on the backing and inspiration from the family members and health care professionals. Some studies on knowledge, attitude, and practices towards breastfeeding are done in rural India [2-5]. This study is aimed to assess the breastfeeding practices and to find out the factors which influence breastfeeding among mothers in a rural area of
South India. Information about breastfeeding practices in the suburban population will be useful for policymakers and for interventional programs.

\section{MATERIALS AND METHODS :}

A descriptive, questionnaire based study was done among mothers with children aged between up to 2 years attending suburban hospitals. Sample size calculation was done as per Third National Family Health Survey approximately $46 \%$ up to 6 months (NHFS-3) report [2]. The sample size of 400 was taken to be accurate. Questionnaires to 400 mothers with children in the age group of up to 2 years. Informed consent was obtained from the mothers of children aged up to 2 years. A faceto-face interview using a pre-designed pretested and validated uniform questionnaire concerning practices of breastfeeding was conducted. The questionnaire included data about the maternal holding of the baby, maternal age, parity, type of delivery, place of delivery, education, employment, socioeconomic status, religion, residence, gender of the child, initiation, and duration of exclusive breastfeeding and complementary feeding, bottle feeding. Conditions, where breastfeeding is contraindicated were excluded from the study. Health education was given to all the mothers interviewed regarding the advantages of breastfeeding.

\section{STATISTICAL ANALYSIS :}

SPSS statistical software (Version 17) was used for data analysis. Statistics like mean, frequency and percentages of various variables were calculated. Chi-Square test and Spearman's Correlation test was used to infer the association and correlation between the period of exclusive breastfeeding with different attributes and $\mathrm{p}$ values were calculated. The $\mathrm{p}$ value $<0.05$ was considered significant and $\mathrm{p}<0.01$ was highly significant.

\section{RESULTS :}

In this study, the majority of the mothers were in the age group 18 to 35 years of age most of them from lower middle class had basic education. Many were hospital deliveries, 20 deliveries conducted at home were conducted by Dai.

Maternal Information regarding of Breast feeding : 
$93.2 \%$ had regular antenatal visits of up to 4 or more. Most of the mothers had antenatal counseling concerning benefits and administration of breastfeeding by doctors and nurses. Information from health personnel prior to conception from family and friends, media or literature and from previous experience was received. It is evident that majority (72.8\%) had knowledge about advantages of breastfeeding that child remains healthy. Very few mothers knew about feeding of twin babies, Fore milk and Hind milk. None of the mothers in this study had knowledge about Milk Bank, Surrogate Mothers or Wet Nursing and Breastshield or Nipple shield. The study shows that most of the mothers had a satisfactory attitude towards breastfeeding. $67.5 \%$ of mothers approved that breast milk is the best milk. Very few mothers felt that breastfeeding is out of fashion reveals practices regarding breastfeeding. It was noticed that opportune suckling in the first hour of birth was practiced only by $43.8 \%$ mothers. 27.0\% mothers given prelacteal feeds to their infants. Prelacteal foods included glucose water, plain water, tea, honey, juice, ginger water and herbal drink. Prelacteal feeds were given by spoon, finger, cloth wick and cotton wick. Colostrum was administered by $88.0 \%$ of mothers. Out of 400 cases, 32.0\% babies were exclusively breastfed. Most mothers breastfed on demand and a small number did scheduled timings. Night feeds were given by all mothers. In this study, most of the mothers burped their infants after every feed and more than two-thirds knew about the hygiene of breast and hand washing before feeds. A small percentage of the mothers used pacifiers.

\section{Newborn data :}

Of the infants and children surveyed 47 children were less than 1 month, 41 were between 1-2 months, 71 were between 3-6 months, 108 were between 7 months-1 year and 133 were between 1-2 years. Out of 400 cases, 29 had neonatal problems among which hyper-bilirubinemia, prematurity and LBW/IUGR were the most common cause. During the period of separation, out of 20 mothers, only 10 had expressed their breast milk and stored in the refrigerator on the advice of doctor whose babies were admitted to the hospital. The mothers were able to preserve the expressed breast milk and fed their babies.

\section{DISCUSSION :}

This study, done in this population revealed that the commonest method of infant feeding was breastfeeding. Infant feeding practices affect child survival and development. Most Indian women start breastfeeding early and breastfeed their children for a long their time. Majority of mothers had delivered in the hospital and nearly two-thirds of them received antenatal advice regarding breastfeeding. 34.5\% mothers had heard some breastfeeding promotional messages from the media. Various studies done on breast feeding campaigns shows that they have a beneficial influence on breast feeding [6-7]. Media is a powerful tool to promote breast feeding in this era. In this study, the breastfeeding pattern, though not optimal as more than two-thirds of mothers initiated breastfeeding within first 4 hours. $\quad 43.8 \%$ of mothers initiated breastfeeding within 1 hour after delivery. By applying the Chi-Square test, the association between the initiation of breastfeed with the mode of delivery was found to be highly significant $(p<0.01)$. Most of the mothers had introduced breastfeeds within one hour after normal vaginal delivery, only a small number started after caesarean section. The delay in initiation of feeds in mothers who delivered by Caesarean section was mainly due to delay in shifting the mother from the labour room to the ward. Hence intensive efforts need to be put for timely initiation of breastfeeding preferably within labour room itself if there is a delay in shifting the mothers. However, at the end of 4 hours $90.57 \%$ of mothers initiated breastfeed after normal vaginal delivery and 82\% after caesarean section. Postponement in the initiation of breastfeeding is seen in others studies [5-8]. The initiation of breast-feeding had no correlation with socioeconomic status, education, sex of the child and parity ( $p>0.05)$. Deferral in initiating breastfeeding affected the amount of breast milk made because of the postponement in the stimulus provided by suckling. Hypoglycemia, hypothermia, and acidosis can occur among low birth weight infants. Rooming in was practiced. Infants who were not roomed in were due to NICU admission due to neonatal problems. Out of 
the 20 babies, only 10 mothers had expressed their breast milk on the advice of a doctor. In our study, prelacteal feeds were given by $27 \%$ of mother contrary to study done by Banapurmath et al [9] which shows $100 \%$ prelacteal feeds usage. Prelacteal feeds are potentially harmful to the newborns as they could introduce infection, sensitize the gut to foreign proteins, or postpone the onset of lactation. Nearly all 88\% infants received colostrum agreeing with a study by Banapurmath et al [9] reported colostrum feeding rate of $71.4 \%$. Benakappa et al [10] showed that colostrum was rejected by $58.4 \%$.Other studies also [11-13] showed that $30 \%$ mothers considered colostrum as not acceptable. By applying Chi-Square test we tried to infer the association between the educational status of the mother with colostrum feeding, it was found to be highly significant $(p<0.01)$. The practice of denial of colostrum was more common in uneducated mothers.

In this study out of 400 cases, 128 were still on exclusive breastfeeding. Among them, half the number continued exclusive breastfeeding for 4 months while other half for 5-6 months which was also noticed by Ekambaram et al [14]. There was no correlation between duration of exclusive breastfeeding with parity, mode of delivery, employment, socioeconomic status and sex of the child. A good correlation with the scholastic status of mothers and initiation of breastfeeding was found evident in our study. The lower the scholastic status of the mother, more the postponement in the initiation of breastfeeding was found. Similar trend is also noticed in western countries [6]. It was observed that duration of exclusive breastfeeding was sustained for a lengthier time in Hindus and Christians compared to Muslims. Bottle-feeds were practiced by $34 \%$ of mothers, gave bottle-feeds in between breastfeeds. Bottlefeeding was more usually practiced by less sophisticated mothers and Muslim mothers. None of the mothers stopped breastfeeding because of mother's ill health. 38.5\% of mothers felt that period of exclusive breastfeeding were satisfactory. (21\%) felt inadequate breast milk production made the babies cry. $10.3 \%$ had stopped because of relative's advice. Only $0.8 \%$ mothers stopped due to unfavorable working conditions. $1 \%$ mothers had stopped because of retracted nipple but later continued with support by doctors to feed the infant by using the syringing technique. Mothers, who gave insufficient breast milk production as a reason, also gave breast rejection by the baby as the following reason, as their babies had been put on supplemental feeds due to inadequate output perceived by the mother and the child had preferred taking top feeds. 1.3\% of mothers stopped because of an ensuing pregnancy. Most mothers had a sound knowledge and a positive attitude towards breastfeeding. $72.8 \%$ mothers had knowledge about advantages of breastfeeding $67.5 \%$ mothers approved that breast milk is the best milk. 29.3\% recognized it is more nutritious and hygienic and there is a fewer chance of infection as it is hygienic and sterile. 20.5\% knew about Lactational Amenorrhea as a natural method of contraception. $75.5 \%$ agreed that it fosters a close bond between the mother and child. Only 5.25\% felt that breastfeeding is old fashioned while it leads to loss of figure was felt by $1.25 \%$ mothers. $4.25 \%$ of mothers felt that breastfeeding in public was embarrassing. Kar and De [15] in their study also noticed similar advantages of breast milk being whole, clean and uncontaminated food for the baby and suitable to the mother as seen by Grovel et al [16] in their study in East Delhi. In a study from Jordan [8] a small percentage of illiterate mothers were shy of feeding in public. The breastfeeding technique was good and Most of the mothers knew to keep nipple and most of the areola inside the mouth of the infant, also about the cleanliness of breast and hand washing before feeds and burping after feeds. Benakappa et al [10] showed that $42 \%$ did not burp their infants. The present study differs from other studies as the majority of deliveries in this study were conducted in the hospital where they were directed by doctors and nurses concerning breastfeeding benefits and its supervision.

In the present study, Top feeds were started by $48.6 \%$ mothers before the conclusion of 6 months of the child. $54.5 \%$ mothers had begancow's milk and $15.5 \%$ had started formula milk before the conclusion of 6 months. Satisfactory supply of human breast milk placates virtually all the nutritional needs 
of an infant for the first 6 months of life. Bottle-feeds were given by $34 \%$ of mothers. The mean age of starting bottle feeds in this study was 4 months; used to give bottle-feeds in between breastfeeds. Bottle-feeding was more commonly practiced by less sophisticated mothers and Muslim mothers. The advice for bottle-feeds was given by relatives (mother in law or grandmother) in $45.82 \%$ of cases while $50.75 \%$ mother herself took the decision and only 1 mother was advised by doctor. There is an urgent need to educate the mothers regarding the disadvantages of bottle-feeding. A study done by Sachdev and Mehrotra [17] showed bottlefeeding rate of $31.5 \%$, similar to the present study. The mean age of starting complementary feeds in this study was 6.5 months, but few mothers initiated complementary feeds much earlier as early as 1 month, mainly due to misconception, customs and pseudo beliefs regarding breastfeeding practices prevalent in this community. Advice for introduction of complementary feeding was obtained from the health personnel, relatives and peer group. Breastmilk alone is not sufficient to satisfy the nutritional needs to sustain optimal growth beyond 6 months. The initial supplementary foods vary from place to place and depend on knowledge, customs and local availability of foods. Apart from increased risk of infection, early introduction of supplementary foods may have a negative effect on breastfeeding and can also affect families belonging to lower socioeconomic class people in terms of cost of food. Most of them used homemade complementary foods but the commercial complementary food was used by families belonging to higher income group. The same trend was also noticed by Banapurmath et al [9] in the villages of central Karnataka and less in a study done [18] in Baroda and in Delhi Slums [19]. Enlightening the mothers regarding usage of cost-effective and easily available home-made complementary foods by using combination of any pulses, cereals and legumes. No gender discrimination was established in this study with regards to commencement of complementary feeds comparable to the study done by Savage et al [20].
One of the significant observations made was that mothers followed stringent food taboos after childbirth. They avoided certain kinds of foods, which according to them were damaging for the infants (green leafy vegetables, lemons, oranges, oily foods, banana, and chillies). The everyday diet of rural women is rarely nutritionally adequate; it becomes less so throughout pregnancy and lactation. The cultural practice of dietary restrictions deprives postpartum and lactating women of some of the more nutritious diet which may disturb the overall health and wellbeing of both mother and her infant. These findings apparently highlight the consequence of educating the women on the importance of good breastfeeding practices and the knowledge may be communicated even at the school or college level.

\section{CONCLUSION :}

There is a need to focus more on teaching the less educated women on breast feeding practices with correct information on good hygienic practices while breastfeeding the child. Efficient orientation programmes on breastfeeding counselling for mother arranged by health care providers to instruct women on breast feeding optimal practices. Women organizations too, need to be linked for the active broadcasting of information on breastfeeding to educationally underprivileged women. It is essential for programmes, which support and boost breastfeeding at a primary level, concentrating more on younger mothers, less well educated and those from lower socioeconomic class.

\section{REFERENCES :}

[1] WHO/breastfeeding. http://www.who.int/e ntity/child_adolescent

health/topics/preventi

child/nutrition/breastfeeding/en. Acc essed on 14 April, 2013.

[2] International Institute for Population Science, Mumbai for Government of India. National Family Health Survey-3 (NFHS-3); 2005-06.

[3] Ramkrishna M.N. A study in breast feeding practices in a rural coal mine area of Andhra Pradesh. Indian J. Public Health. 2000; 44: 65-6. 
[4] Shalini C, Chakladar B.K., Rao R.S.P. Infant feeding- Knowledge and attitudes in a rural area of Karnataka. Indian J. Pediatr 1995; 62: 707-712.

[5] Kapil V, Kaul S, Vohra G, Chaturvedi S. Breastfeeding practices among mothers having undergone Caesarean section. Indian Pediatr, 1992; 29: 222-224.

[6] Earle S. Factors affecting the initiation of breastfeeding: implications for breastfeeding promotion. Health PromotInt, 2002;17:205- 214.

[7] Haider R, Kabir I, Ashworth A. News from the region. Are breastfeeding promotion messages influencing mothers in Bangladesh. Results from an urban survey in Dhaka. Bangladesh. J. Trop Paediatrics, 1999;45:315- 318.

[8] Khassawneh M, Khader Y, Amarin Z, Alkafajei A. Knowledge ,attitude and practice of breast feeding in the north of Jordan; a cross sectional study. Int. Breast-feeding J. 2006; 17.

[9] Banapurmath C. R., Nagaraj M. C., Banapurmath S., Kesaree N. Breastfeeding practices in villages of central Karnataka. Indian Pediatr 1996; 33:477-479.

[10] Benakappa A. V, Raja D. G, Acta M. Breastfeeding practices in rural Karnataka (India) with special reference to lactation failure. Paediatr Jpn 1989 ; 31:391-398.

[11] Kumar S, Nath L. M, Reddy V.P. Breastfeeding practices in a resettlement colony and its implications for promotional activities. Indian Pediatr 1989; 56: 239-42.

[12] Malireddy R, Sekhar K.C. Knowledge, attitude, practice regarding breast feeding practice among mothers attending. Ind J. Pub Health Develop 2012; 3:76-79.

[13] Yadav R. J, Singh P. Knowledge, Attitude and Practices of Mothers about BreastFeeding in Bihar. Ind J. Comm Med 2004; 29:130-133.

[14] Ekambaram M, Bhat V.B., Ahamad M.A.P. Knowledge, attitude and practice of breast-feeding among postnatal
Mothers. Curr Pediatr Res 2010;14: 119124.

[15] Kar M, De R. Breast Feeding Practices Impressions from an Urban Community. Ind J. Pub Hlth. 1991; 35: 93-97.

[16] Grover V.L, Chhabra P, Aggarwal O.P. Knowledge, Attitude and Practices of Breast Feeding In A Rural Area of East Delhi. Health and PopulationPerspectives and Issues 1993; 20: 49-56.

[17] Sachdev H.P.S, Mehrptra S. Predictors of exclusive breastfeeding in early infancy: operational implications. Indian Pediatr 1995; 32.1287-92.

[18] Pant I, Chothia K. Maternal knowledge regarding breast feeding and weaning practices. Ind J. Ped 1990; 57: 395-400

[19] Khokar A, Singh S, Talwar R et al. A study of malnutrition among children aged 6 to 24 months from a resettlement colony in Delhi. Ind J Med Sci. 2003; 57: 287-289.

[20] Savage Shirley AH, Reilly JJ, Edmonds CA, Durnin John VGA. Weaning practice in the Glasgow longitudinal infant growth study. Arch Disease Child1988; 79: 153156. 